\title{
PDMS composites with photostable NIR dyes for multi-modal ultrasound imaging
}

\author{
India Lewis-Thompson ${ }^{1,2} \cdot$ Shaoyan Zhang ${ }^{1,2} \cdot$ Sacha Noimark ${ }^{1,2,3} \cdot$ Adrien E. Desjardins $^{1,2} \cdot$ Richard J. Colchester $^{1,2}$ (1)
}

Received: 17 November 2021 / Accepted: 27 December 2021 / Published online: 18 January 2022

(c) The Author(s) 2022

\begin{abstract}
All-optical ultrasound (OpUS) imaging has emerged as an imaging paradigm well-suited for minimally invasive surgical procedures. With this modality, ultrasound is generated when pulsed or modulated light is absorbed within a coating material. By engineering wavelength-selective coatings, complementary imaging and therapeutic modalities can be integrated with OpUS. Here, we present a wavelength-selective composite material comprising a near-infrared absorbing dye and polydimethylsiloxane. The optical absorption for this material peaked in the vicinity of $1064 \mathrm{~nm}$, with up to $91 \%$ of incident light being absorbed, whilst maintaining lower optical absorption at other wavelengths. This material was used to generate ultrasound, demonstrating ultrasound pressures $>1 \mathrm{MPa}$, consistent with those used for imaging applications. Crucially, long exposure photostability and device performance were found to be stable over a one hour period (peak pressure variation $<10 \%$ ), longer than required for standard clinical imaging applications.
\end{abstract}

\section{Introduction}

All-optical ultrasound imaging has emerged as an imaging paradigm well-suited for minimally invasive surgical procedures [1-3]. With this modality, ultrasound is generated when pulsed or modulated light is absorbed within a composite coating material [4-8]. Using optical fibres to deliver

Richard J. Colchester

richard.colchester@ucl.ac.uk

India Lewis-Thompson

india.thompson.20@ucl.ac.uk

Shaoyan Zhang

shaoyan.zhang.20@ucl.ac.uk

Sacha Noimark

s.noimark@ucl.ac.uk

Adrien E. Desjardins

a.desjardins@ucl.ac.uk

1 Department of Medical Physics and Biomedical Engineering, University College London, Gower Street, London WC1E 6BT, UK

2 Wellcome/EPSRC Centre for Interventional and Surgical Sciences, University College London, Charles Bell House, Foley Street, London W1W 7TY, UK

3 Materials Chemistry Centre, Department of Chemistry, University College London, 20 Gordon Street, London WC1H 0AJ, UK light for ultrasound generation and reception allows complementary therapeutic and imaging modalities to be integrated to create highly miniaturised devices $[1,4,9,10]$. One area of interest for these devices is combined imaging and optical ablation, to provide real-time monitoring of ablation lesion depth. However, combining modalities requires carefully engineered composite materials with wavelength-selective absorption $[1,4,9,10]$. Previous research has demonstrated the use of crystal violet dye or gold nanoparticles embedded in a polydimethylsiloxane (PDMS) host for co-registered ultrasound and photoacoustic imaging [1]. However, these composites suffered from poor photostability under prolonged laser exposure and limited ultrasound pressures and bandwidths, respectively. More recently, quantum dots have been used for co-registered ultrasound and photoacoustic imaging, but the quantum dots were synthesised through a complex process [10].

In this work we introduce a composite comprising a nearinfrared absorbing dye and PDMS. The dye (Epolight 9837, Epolin, USA) selected was recommended for absorption of $1064 \mathrm{~nm}$ laser light and demonstrates high optical transmission in the visible range when embedded in polycarbonate [11]. This wavelength-selective nature is highly desirable for combining complementary modalities with all-optical ultrasound imaging. Here, we combined the dye with PDMS to fabricate ultrasound generating coatings on optical fibres. Three composites with varying dye concentrations were 
fabricated to assess the optimal dye concentration; for each concentration 5 ultrasound transmitter repeats were carried out to assess variability. The optical absorption, generated ultrasound, and photostability were characterised and compared to a composite of crystal violet (CV) and PDMS for reference.

\section{Methods}

\section{Fabrication}

Four composite coatings were fabricated; three using Epolight 9837 with varying concentration of dye by weight $(2.5$, $5,10 \mathrm{mg}$ ) and one using CV for comparison as previous studies show this dye is susceptible to photobleaching [1]. For each composite coating, 5 ultrasound generators were fabricated to test for consistency. All composite coatings were fabricated on the same type of optical fibre, with a fused silica core/cladding and a core diameter of $400 \mu \mathrm{m}$ (FG400LEP, Thorlabs, UK). The CV fibres were manufactured as per the protocol outlined by Noimark et al. [1]. For the Epolight composites, the selected weight of dye was sonicated at $40 \mathrm{~W}$ for $30 \mathrm{~s}$ in $0.5 \mathrm{ml}$ of xylene until the dye was dissolved. Subsequently, this solution was manually mixed with $0.25 \mathrm{~g}$ of PDMS (MED-1000, Polymer Systems
Technology, UK), followed by sonification at $30 \mathrm{~W}$ for a total of $30 \mathrm{~s}$, in three $10 \mathrm{~s}$ periods with a $10 \mathrm{~s}$ rest between.

Optical fibres were prepared for coating by first removing the buffer coating from the distal end of the fibre. Subsequently, the distal end was cleaved manually using a tungsten blade. The prepared optical fibres were manually dipped into the prepared composite solutions and left to cure under ambient conditions for 24 hours with the distal end surface facing up.

\section{Characterisation}

Prior to optical and ultrasound characterisation, the coated optical fibres were examined visually using a stereo-microscope (Fig. 1a-c).

The wavelength-dependent optical absorption for the dye-PDMS coated fibres was measured between 400 and $1700 \mathrm{~nm}$ using a halogen lamp, an integrating sphere, and two spectrometers (Flame (400-700 nm) \& NIRQuest512 (700-1700 nm), Ocean Optics, USA). An uncoated optical fibre was used for reference and a background count was taken for each measurement to remove ambient light and dark counts.

The ultrasound generation was characterised at a pulse energy of $20 \mu \mathrm{J}$ (corresponding fluence at coating: $15.9 \mathrm{~mJ} / \mathrm{cm}^{2}$ ) using two separate pulsed Nd:YAG lasers for
Fig. 1 Stereo-microscope images of a $2.5 \mathrm{mg}$ Epolight composite coated optical fibre. (a) End-view with through illumination. (b) End-view with no through illumination. (c) Side-view. Scale bar: $400 \mu \mathrm{m}$. (d) Optical absorption spectra for the crystal violet (blue solid line) and Epolight composites ( $2.5 \mathrm{mg}$ : red dotted line, $5 \mathrm{mg}$ : red dashed line, $10 \mathrm{mg}$ : red solid line).
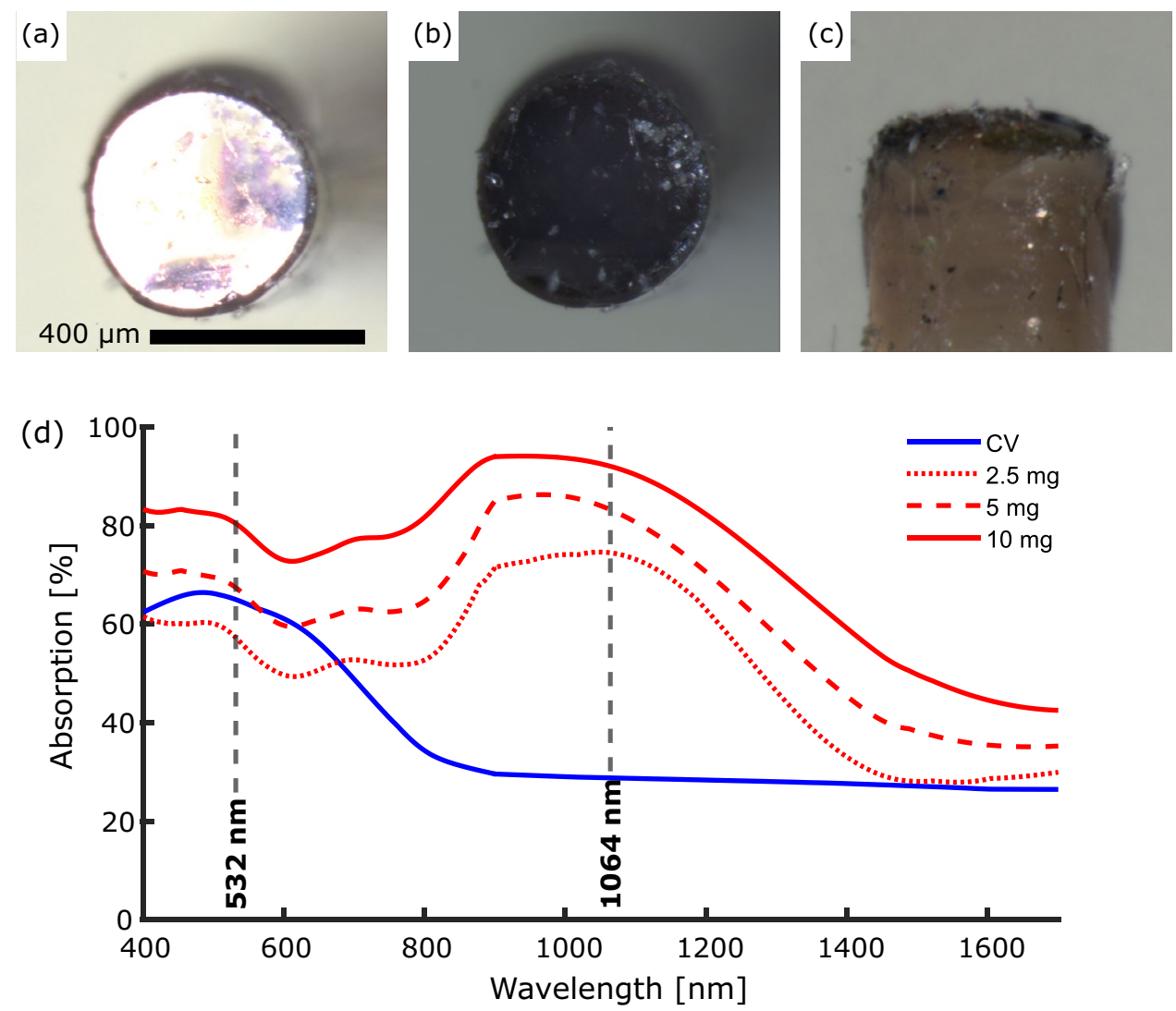
excitation; one with a wavelength of $1064 \mathrm{~nm}$ (pulse width: 2 ns, repetition rate: $100 \mathrm{~Hz}$, Spot-10-500-1064, Elforlight, $\mathrm{UK}$ ), and another with a wavelength of $532 \mathrm{~nm}$ (pulse width: $10 \mathrm{~ns}$, repetition rate: $100 \mathrm{~Hz}, \mathrm{FQ}-500-532$, Elforlight, UK). The generated ultrasound was measured at a distance of $1.5 \mathrm{~mm}$ from the coating using a $200 \mu \mathrm{m}$ needle hydrophone (Precision Acoustics, UK) with a calibration range 1-30 MHz (Fig. 2a)). To obtain the ultrasound bandwidth, the acquired time-series were Fourier transformed and the hydrophone calibration was applied. To measure the photostability, coatings were continuously exposed to laser pulses for a period of 2 hours and an ultrasound measurement was recorded every 10 seconds.

\section{Results}

\section{Optical absorption}

The CV and Epolight dyes exhibited different optical absorption profiles (Fig. 1d)). The Epolight composites exhibited an optical absorption peak at $c a .900-1100 \mathrm{~nm}$ for all dye concentrations (Table 1, Fig. 1d)) with a maximum of $>90 \%$ for the composite with the highest concentration of dye $(10 \mathrm{mg})$. For wavelengths shorter than $900 \mathrm{~nm}$ and longer than $1100 \mathrm{~nm}$ the optical absorption decreased. The optical absorption at all wavelengths decreased with decreasing dye concentration for the Epolight composites. The CV composite exhibited an absorption peak at $c a .532 \mathrm{~nm}$ with a value $c a$. 66\%. The optical absorption decreased for longer wavelengths to a value $<30 \%$.

\section{Ultrasound generation}

The Epolight composites exhibited peak-to-peak ultrasound pressure with values up to $1 \mathrm{MPa}$, with corresponding $-6 \mathrm{~dB}$ ultrasound bandwidths $c a .20 \mathrm{MHz}$ (Fig. 2b-f). The generated ultrasound pressure was found to increase with increasing dye concentration from $2.5 \mathrm{mg}$ to $5 \mathrm{mg}$ with maximum values of 0.46 and $1.03 \mathrm{MPa}$, respectively. For the highest dye concentration of $10 \mathrm{mg}$, the peak-to-peak pressure was found to decrease slightly, with a value of $0.92 \mathrm{MPa}$. The
$\mathrm{CV}$ composite used for reference generated lower peak-topeak pressure and narrower bandwidths (Table 1, Fig. 2b, f).

\section{Photostability}

The photostability of the Epolight composites was compared to a CV composite (Fig. 2g)). With a laser wavelength of $532 \mathrm{~nm}$ at a pulse energy of $20 \mu \mathrm{J}$, the peak-to-peak pressure generated by the CV composite fell by $68 \%$ in 1 hour, compared to a reduction by $7 \%$ for a $10 \mathrm{mg}$ Epolight composite with a similar optical absorption at $532 \mathrm{~nm}$. All Epolight concentrations demonstrated good photostability at $1064 \mathrm{~nm}$, with the pressure dropping by $25 \%, 15 \%$ and $10 \%$ for the $2.5,5$ and $10 \mathrm{mg}$ composites, respectively.

\section{Discussion and conclusion}

In this work we demonstrate the fabrication of a composite comprising an Epolight dye and PDMS and compare it to a CV and PDMS composite. It was found that by reducing the concentration of the Epolight dye, the optical absorption in the range 400 to $800 \mathrm{~nm}$ and for wavelengths $>1200 \mathrm{~nm}$ could be reduced whilst maintaining a relatively high optical absorption at $1064 \mathrm{~nm}$ for ultrasound generation. The lower optical absorption at wavelengths from 400 to $900 \mathrm{~nm}$ and $>1100 \mathrm{~nm}$ is well-suited to a variety of complementary modalities, including photoacoustic imaging and optical ablation. However, whilst the presence of these optical absorption windows are promising for multimodality imaging, further optimisation is needed to reduce the absorption on either side of the peak at $1064 \mathrm{~nm}$. This will be explored in the future work by testing lower concentrations and alternative fabrication methods, such as the bottom-up approach highlighted in [1].

The ultrasound generated by the composites was comparable to that used in previous studies for imaging $[2,3$, 8]. All the Epolight composites fabricated outperformed the reference $\mathrm{CV}$ composite fabricated here, exhibiting a peak-to-peak pressure $>1 \mathrm{MPa}$ for composites with $5 \mathrm{mg}$ of dye. It should be noted, however, that previous studies with CV composites have achieved higher pressures and bandwidths [1]. Composites with $2.5 \mathrm{mg}$ generated lower ultrasound pressures than the $5 \mathrm{mg}$ composites, as expected
Table 1 Optical absorption and ultrasound generation properties of the composite coatings

\begin{tabular}{lllllll}
\hline & \multicolumn{2}{l}{ Optical Absorption [\%] } & & \multicolumn{2}{l}{ Ultrasound Characteristics } \\
\cline { 2 - 3 } & $@ 1064 \mathrm{~nm}$ & @ 532 nm & @ $400-800 \mathrm{~nm}$ & & Pressure [MPa] & Bandwidth [MHz] \\
\hline Crystal violet & $29 \pm 11$ & $66 \pm 14$ & $51 \pm 15$ & & $0.31 \pm 0.02$ & $10.6 \pm 1.6$ \\
$2.5 \mathrm{mg}$ Epolight & $78 \pm 4$ & $67 \pm 7$ & $65 \pm 8$ & & $0.46 \pm 0.14$ & $16.0 \pm 2.7$ \\
$5 \mathrm{mg}$ Epolight & $86 \pm 2$ & $73 \pm 5$ & $72 \pm 4$ & & $1.03 \pm 0.13$ & $20.7 \pm 3.3$ \\
$10 \mathrm{mg}$ Epolight & $96 \pm 2$ & $86 \pm 7$ & $86 \pm 6$ & & $0.92 \pm 0.17$ & $20.5 \pm 2.3$ \\
\hline
\end{tabular}


Fig. 2 (a) Schematic of the ultrasound characterisation setup with the input laser pulse (pulse width: $2 \mathrm{~ns}$ ) and the output ultrasound signal. (b)-(e)

Ultrasound time-series for the composite coatings (CV coating: blue solid line, Epolight coatings: $2.5 \mathrm{mg}$-red dotted line, $5 \mathrm{mg}$-red dashed line, $10 \mathrm{mg}$ red solid line). (f) Corresponding ultrasound power spectra for the composite coatings. (g) Normalised photostability for the composites compared to the crystal violet composite. (a)

water bath

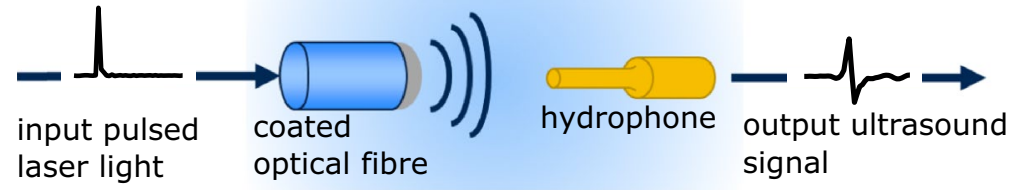

(b)

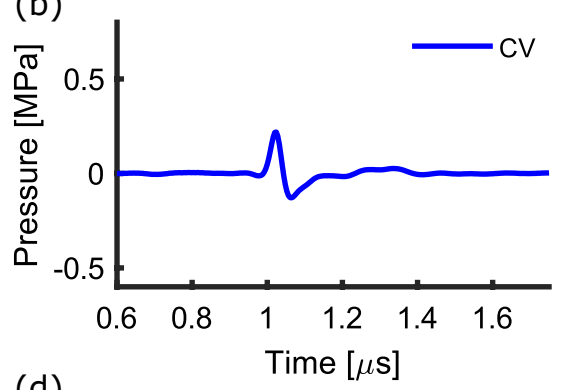

(c)

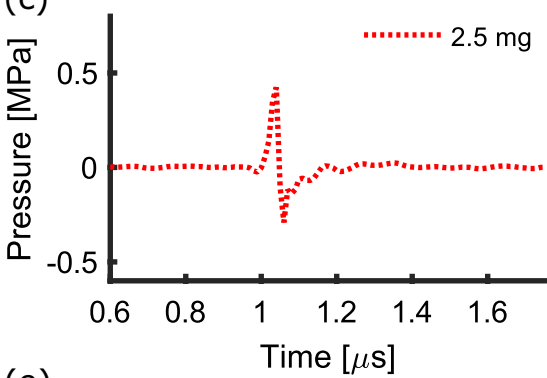

(d)

(e)
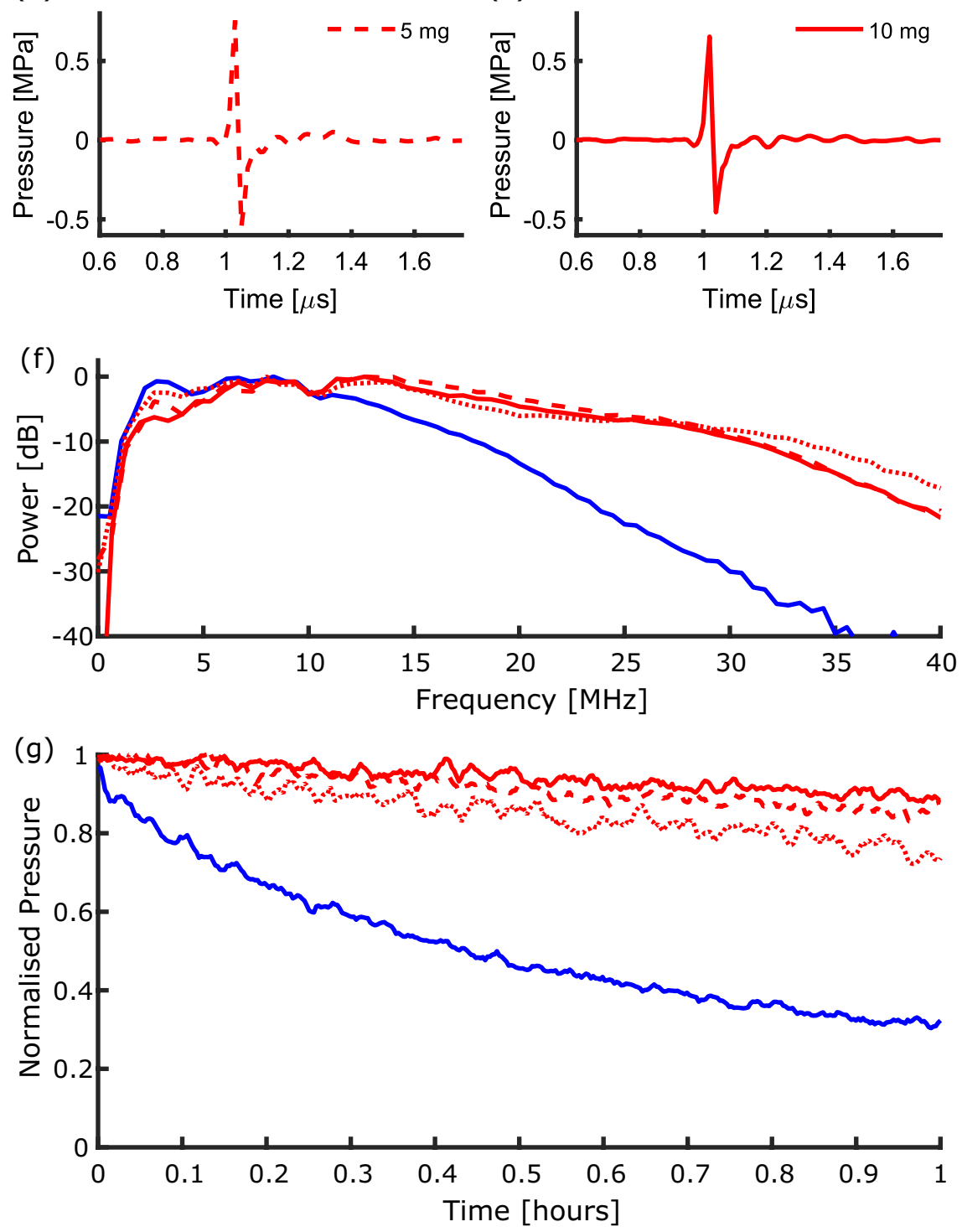
from the corresponding decrease in optical absorption. However, for the higher dye concentration of $10 \mathrm{mg}$, the peak-to-peak pressure was found to decrease. This may be due to increased ultrasound attenuation within the coating caused by the higher dye loading. Ultrasound bandwidths up to $20 \mathrm{MHz}$ were achieved, sufficient for high resolution ultrasound imaging. However, the lower dye concentration of $2.5 \mathrm{mg}$ exhibited narrower bandwidths than other composites. This could be caused by the longer absorption length, leading to a wider temporal profile in the generated ultrasound pulse.

Crucially, the photostability of the Epolight composites was measured and compared to the CV composite, which has previously been used for ex vivo imaging, but demonstrated poor photostability [1]. All of the Epolight composites had acceptable photostability, with the pressure only dropping by $c a .10 \%$ over a period of 1 hour, compared to a $68 \%$ drop for $\mathrm{CV}$ composites over the same period. These timescales are relevant for translation of the devices to minimally invasive imaging contexts, where procedures typically take $>1 \mathrm{~h}$. The results here demonstrate that composite coatings comprising Epolight 9837 are promising for application in multimodality all-optical ultrasound devices. Further work will be carried out to optimise the coatings for specific applications and further improve the generated ultrasound properties. In the future studies, other dyes might be considered with different wavelength selectivities, allowing for application-specific composites. However, for each dye, considerations including the ease of incorporation into the PDMS host, ultrasound generation efficiency and photostability will need to be carefully optimised.

Acknowledgments This research was supported by the Royal Academy of Engineering under the Research Fellowship Scheme (RF/201819/18/125), the Wellcome/EPRSC Centre for Interventional and Surgical Sciences $(203145 / Z / 16 / Z)$, the EPSRC UKRI innovation fellowship Grant (EP/S001506/1), and the EPSRC-funded UCL Centre for Doctoral Training in Intelligent, Integrated Imaging in Healthcare (i4health) (EP/S021930/1).

Data availability The datasets generated during and/or analysed during the current study are available from the corresponding author on reasonable request.

\section{Declarations}

Disclosures RJC \& AED own shares in Echopoint Medical Ltd.

Open Access This article is licensed under a Creative Commons Attribution 4.0 International License, which permits use, sharing, adaptation, distribution and reproduction in any medium or format, as long as you give appropriate credit to the original author(s) and the source, provide a link to the Creative Commons licence, and indicate if changes were made. The images or other third party material in this article are included in the article's Creative Commons licence, unless indicated otherwise in a credit line to the material. If material is not included in the article's Creative Commons licence and your intended use is not permitted by statutory regulation or exceeds the permitted use, you will need to obtain permission directly from the copyright holder. To view a copy of this licence, visit http://creativecommons. org/licenses/by/4.0/.

\section{References}

1. S. Noimark, R.J. Colchester, R.K. Poduval, E. Maneas, E.J. Alles, T. Zhao, E.Z. Zhang, M. Ashworth, E. Tsolaki, A.H. Chester et al., Polydimethylsiloxane composites for optical ultrasound generation and multimodality imaging. Adv. Funct. Mater. 28(9), 1704919 (2018)

2. R.J. Colchester, C. Little, G. Dwyer, S. Noimark, E.J. Alles, E.Z. Zhang, C.D. Loder, I.P. Parkin, I. Papakonstantinou, P.C. Beard et al., All-optical rotational ultrasound imaging. Sci. Rep. 9(1), 1-8 (2019)

3. M.C. Finlay, C.A. Mosse, R.J. Colchester, S. Noimark, E.Z. Zhang, S. Ourselin, P.C. Beard, R.J. Schilling, I.P. Parkin, I. Papakonstantinou et al., Through-needle all-optical ultrasound imaging in vivo: a preclinical swine study. Light 6(12), 17103-17103 (2017)

4. D. Cui, Z. Zhang, Y. Shi, Optically-excited simultaneous photoacoustic and ultrasound imaging based on a flexible gold-PDMS film. J. Innov. Opt. Health Sci. 13(04), 2050012 (2020)

5. S. Noimark, R.J. Colchester, B.J. Blackburn, E.Z. Zhang, E.J. Alles, S. Ourselin, P.C. Beard, I. Papakonstantinou, I.P. Parkin, A.E. Desjardins, Carbon-nanotube-PDMS composite coatings on optical fibers for all-optical ultrasound imaging. Adv. Funct. Mater. 26(46), 8390-8396 (2016)

6. D. Thompson, J.R. Nagel, D.B. Gasteau, S. Manohar, Laserinduced ultrasound transmitters for large-volume ultrasound tomography. Photoacoustics 25, 100312 (2021)

7. T. Lee, J.G. Ok, L.J. Guo, H.W. Baac, Low f-number photoacoustic lens for tight ultrasonic focusing and free-field micro-cavitation in water. Appl. Phys. Lett. 108(10), 104102 (2016)

8. X. Zou, N. Wu, Y. Tian, X. Wang, Broadband miniature fiber optic ultrasound generator. Opt. Express 22(15), 18119-18127 (2014)

9. B.-Y. Hsieh, S.-L. Chen, T. Ling, L.J. Guo, P.-C. Li, All-optical transducer for ultrasound and photoacoustic imaging by dichroic filtering. In: 2012 IEEE International Ultrasonics Symposium, pp. 1410-1413. IEEE (2012)

10. S. Bodian, R.J. Colchester, T.J. Macdonald, F. Ambroz, M. Briceno de Gutierrez, S.J. Mathews, Y.M.M. Fong, E. Maneas, K.A. Welsby, R.J. Gordon et al., Cuins2 quantum dot and polydimethylsiloxane nanocomposites for all-optical ultrasound and photoacoustic imaging. Adv. Mater. Interfaces 8, 2100518 (2021)

11. Epolin: Epolight ${ }^{\mathrm{TM}} 9837$ NIR Dye Technical Data Sheet. https:// www.epolin.com/wp-content/uploads/2021/02/9837-data-sheet. pdf. Accessed 1 November 2021 (2013) 